\title{
Leben mit Knochen wie aus Glas
}

\section{Therese Stutz Steigera ${ }^{a}$ Bérengère Rozier Aubry}

a Ärztin, Präsidentin der Schweizerischen Vereinigung Osteogenesis Imperfecta (SVOI-ASOI) 2011-2020; Vizepräsidentin ProRaris;

${ }^{b}$ Rheumatologin und Expertin für die Betreuung von Patienten mit seltenen Knochen- und Bewegungsapparats-Erkrankungen, CHUV, Lausanne

\begin{abstract}
Im letzten November fand in Amsterdam eine internationale Konferenz zur Glasknochenkrankheit statt, die sich von den wissenschaftlichen Osteogenesis-imperfecta-Kongressen unterschied: Die Konferenz war offensichtlich darauf angelegt, für Fachpersonen und für Betroffene gleichermassen interessant zu sein.
\end{abstract}

Tatsächlich war schon an der Anzahl und Vielfalt der Rollstühle und ähnlicher Fortbewegungsmittel zu erkennen, dass sich unter den 300 Teilnehmenden eine ansehnliche Anzahl Osteogenesis imperfecta (OI)-Patientinnen und -Patienten jeglichen Alters und verschiedenster Ausprägungen des Bindegewebe-Defekts befand. Initiantin und Organisatorin war die Stiftung "Care4BrittleBones» unter der befeuernden Leitung von Dagmar Mekking, die ein ausgezeichnetes Netzwerk zu Betroffenen und OI-Experten aufgebaut hat.

Teilnehmende aus der Schweiz waren Dr. Bérengère Rozier Aubry vom CHUV Lausanne, Dr. Therese Stutz Steiger, Präsidentin Schweizerische Vereinigung Osteogenesis Imperfecta (SVOI-ASOI) a.i., Mark Steiger als Begleiter sowie der Vater eines OI-betroffenen Knaben aus Zürich.

Die Konferenz fand im RAI-Tagungszentrum statt, das nur auf den ersten Blick überdimensioniert schien, tatsächlich aber den Rollstuhlfahrenden oder an Stöcken Gehenden nicht das Gefühl vermittelte, eingeengt zu sein. Auch in den Pausen waren Getränke und kleine Häppchen ohne nennenswerte Wartezeiten erreichbar.

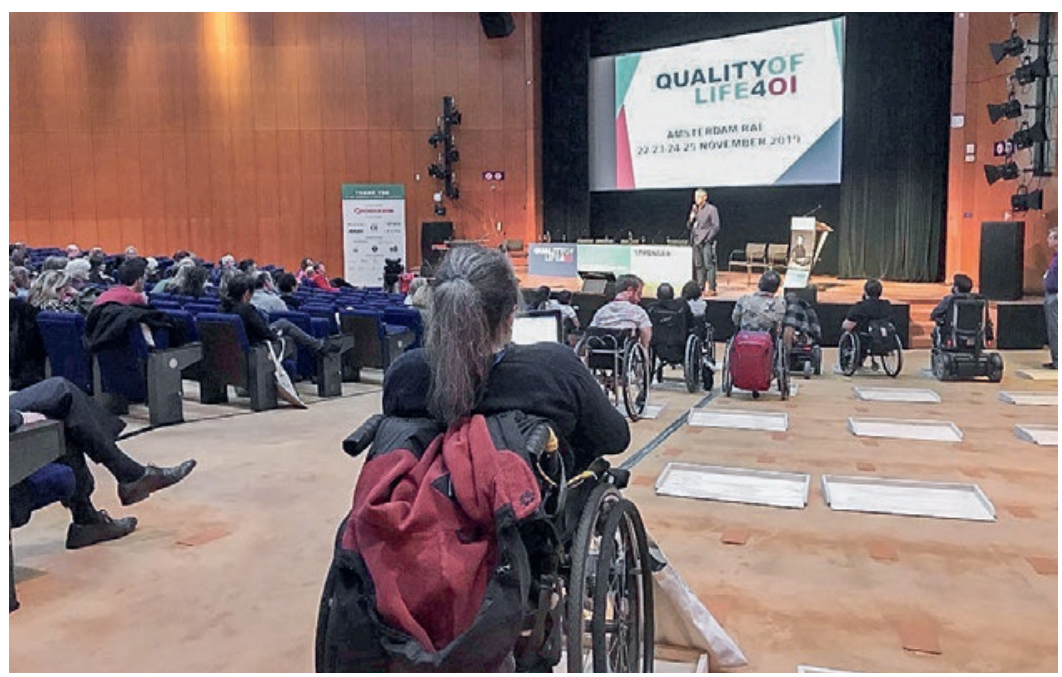

Der Konferenzsaal war mit provisorischen baulichen Massnahmen für Rollstuhlfahrende besonders bequem eingerichtet worden.
Der Konferenzsaal war mit provisorischen baulichen Massnahmen für Rollstuhlfahrende besonders bequem eingerichtet worden.

\section{Gleichzeitig Betroffene und Fachperson}

OI-Betroffene, zum Teil in der Doppelrolle als Patienten und Fachpersonen, setzten auch wesentliche inhaltliche Akzente. Die Konferenzteilnehmer, Patienten, Betreuer und Vertreter von Patientenverbänden, waren deutlich aktiver als bei herkömmlichen Kongressen.

Nebst den Hauptreferaten und Postersessions waren die thematischen «Deep Diving Sessions» ein wichtiger Teil des Programms. Sie wurden parallel geführt; die Betroffenen kamen darin ausführlich zu Wort. Auch an den Postersessions bot sich mannigfach Gelegenheit zum persönlichen Austausch, ebenso natürlich am Conference Dinner, an dem einige meist junge Betroffene erstaunliche künstlerische und Entertainer-Begabungen entfalteten.

Offensichtlich war das Bemühen, OI bei Kindern und bei Erwachsenen gleichberechtigt $\mathrm{zu}$ behandeln und vor allem Aspekte zu berücksichtigen, die sich auf Leben und Lebensqualität der Betroffenen auswirken. Auch sollte den anwesenden Betroffenen und Professionellen die Idee vermittelt werden, dass der Umgang mit OI, insbesondere die Bemühungen der Forschenden und der klinisch bzw. pflegerisch Tätigen, noch vermehrt partnerschaftlich angegangen werden kann. Ein wichtiger Teil des Programms waren die «Deep Diving Sessions» zu den Themen:

- Medikamentöse Therapie: Bisphosphonate bis hin zu Stammzelltherapie

- Orthopädie: Road map to surgery for optimal patient satisfaction

- Diagnostik: Vergangene, aktuelle und künftige Trends (mit Schwerpunkt Genetik)

- Psychosoziale Aspekte: Messmöglichkeiten von Lebensqualität 
- Empowerment: Erst nach einem solchen Prozess können Patienten als Partner auf Augenhöhe auftreten.

\section{OI als systemische Erkrankung}

Weitere Schwerpunkte der Tagung war etwa die neue Rolle des Patienten: Unter dem Titel «The changing role of the patient» hielt Ingunn Westerheim, die Präsidentin der OIFE, des europäischen Dachverbands der nationalen OI-Patientenorganisationen, einen eindrücklichen Vortrag. Sie zeigte auf, dass die Bedürfnisse der Patienten im Spital mit denen der chronisch Betroffenen im Alltag nicht vergleichbar sind.

Von hoher Aktualität waren Berichte über das Europäische Referenznetzwerk ERN BOND, aus dem die Schweiz als Nicht-EU-Mitglied ausgeschlossen ist. Grossbritannien hat hierzu sehr viel beigetragen. Was nach dem Brexit passieren wird, ist aber noch unklar.

Grossen Anklang fand ein Referat über das neu entwickelte Key4OI-Set. Key4OI ist eines der wichtigsten gemeinschaftlichen Projekte im Bereich von OI mit Experten aus mehreren Ländern aus der ganzen Welt. 25\% der Experten haben selber OI. In den letzten zwei Jahren haben sie die wichtigsten Gesundheitsthemen von Menschen mit OI erarbeitet. Dies geschah unter Berücksichtigung aller verschiedenen OI-Typen. Es wurde weltweit diskutiert, wie die Resultate von Behandlung gemessen werden sollen. So wollen Ärzte und Wissenschaftler neue Erkenntnisse zur Effektivität der Behandlung von OI gewinnen.

Relativ neu ist der Ansatz, OI als systemische Erkrankung zu verstehen. Es wurden die Auswirkungen der Krankheit auf den gesamten Körper thematisiert: knochen- und nicht knochenspezifische Aspekte, wie Herzklappen und -scheidewände, Gefässe, Augenhornhaut, Knorpel, Zähne wurden diskutiert.

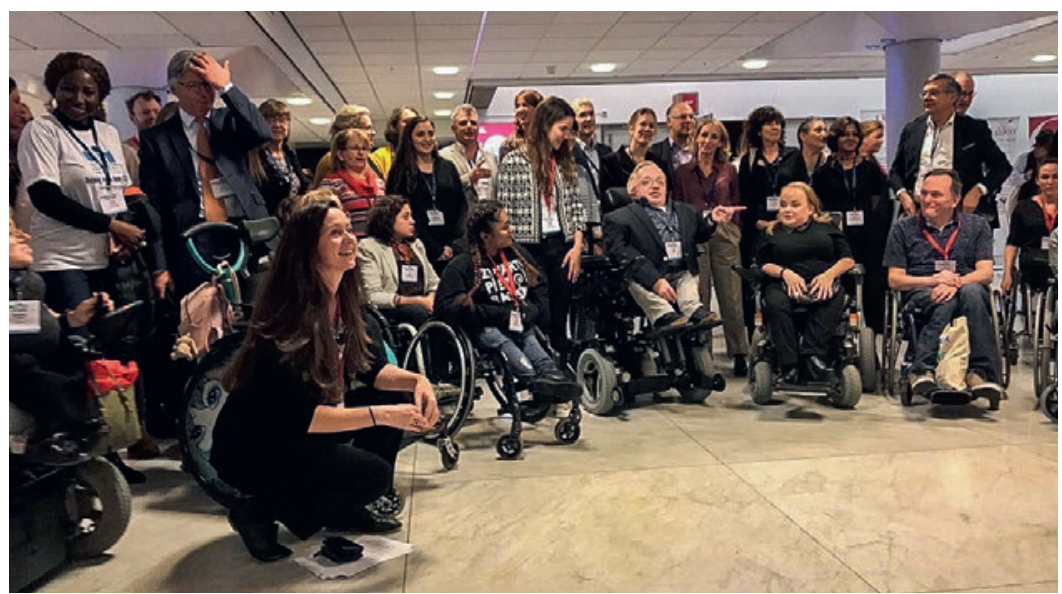

Unter den 300 Teilnehmenden befand sich eine ansehnliche Anzahl Osteogenesis-imperfectaBetroffene jeglichen Alters und verschiedenster Ausprägungen des Bindegewebe-Defekts.

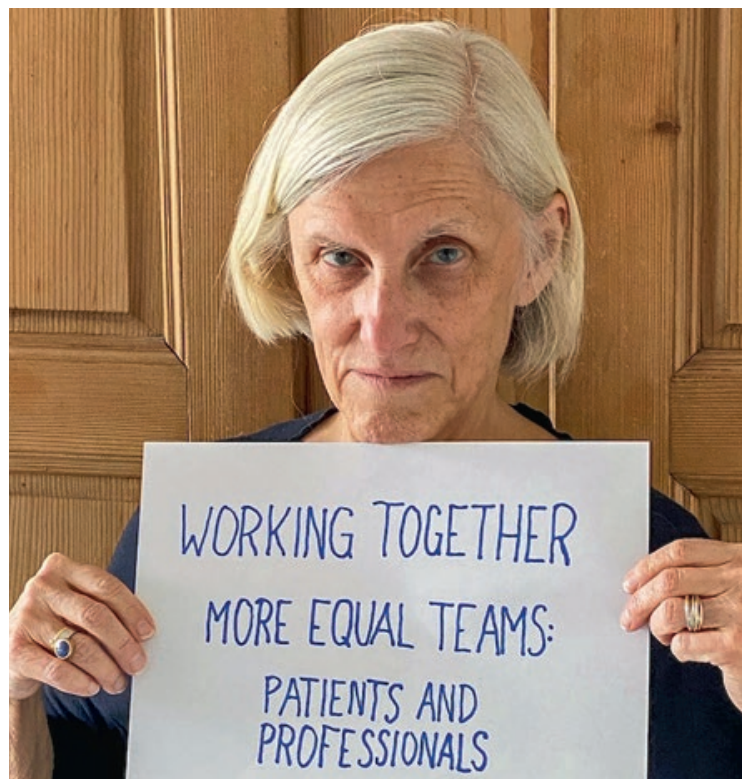

Therese Stutz Steiger ist selber von OI betroffen. Sie äussert im Hinblick auf den "Wishbone Day" am 6. Mai 2020 zusammen mit 60 Betroffenen aus aller Welt ihren Wunsch.

\section{Austausch weiterverfolgen}

Es gibt immer mehr Hinweise, dass für OI-Betroffene und ihre Angehörigen die psychische Belastung sehr hoch ist. Viele müssen sich mit Ängsten und Depressionen auseinandersetzen. Das wurde in der Konferenz insbesondere in den Deep Dive Sessions «Leben mit OI» und «Empowerment» behandelt. Es gibt auf diesem Gebiet noch viel zu tun. Care4BrittleBones wird eine internationale Arbeitsgruppe ins Leben rufen, die verschiedene Themen in diesem Spektrum weiter vertieft und ausarbeitet.

Dialog und Interaktion zwischen allen Beteiligten aus Forschung, Klinik und Pflege sowie Patientenorganisationen konnten gepflegt werden, was seither auch die Kontakte auf den Social Media verstärkt hat. Es bleibt zu hoffen, dass die an der Tagung erfolgten positiven Impulse durch eine vergleichbare Veranstaltung wiederaufgenommen und weiterverfolgt werden und dass noch näher an den Alltag der OI-Betroffenen herangerückt werden kann. Zurück in der Schweiz, versuchen wir die Netzwerkarbeit und die Zusammenarbeit insbesondere mit den Orthopäden und Orthopädinnen zu verstärken.

\section{Dank}

Wir verdanken die Mitarbeit von Mark Steiger (Angehöriger) und Cornelia Jungo (Mitglied SVOI-ASOI).

\section{Bildnachweis}

Therese Stutz Steiger, Mark Steiger

Weitere Informationen zu Osteogenesis imperfecta finden sich unter www.glasknochen.ch 